\title{
The Italian Society for Rheumatology recommendations for the management of axial spondyloarthritis
}

\author{
M. Manara',2, I. Prevete ${ }^{1,3}$, A. Marchesoni', S. D’Angelo ${ }^{4}$, A. Cauli, \\ A. Zanetti ${ }^{1,6}$, A. Ariani $i^{1,7}$, Alessandra Bortoluzzi ${ }^{1,8}$, Simone Parisi $i^{1,9}$, \\ Carlo Alberto Scirè ${ }^{1,8}$, Nicola Ughi ${ }^{1,10}$
}

${ }^{1}$ Epidemiology Research Unit, Italian Society for Rheumatology, Milan, Italy; '2Department of Rheumatology, ASST Gaetano Pini-CTO, Milan, Italy; ${ }^{3}$ Rheumatology Unit, Azienda Ospedaliera San Camillo-Forlanini, Rome, Italy; ${ }^{4}$ Rheumatology Institute of Lucania (IReL) and Rheumatology Department of Lucania, San Carlo Hospital of Potenza, Italy; ${ }^{5}$ Rheumatology Unit, Department of Medical Sciences and Public Health, AOU and University of Cagliari, Italy; ${ }^{6}$ Department of Statistics and Quantitative Methods, Division of Biostatistics, Epidemiology and Public Health, University of Milano-Bicocca, Milan, Italy; ${ }^{7}$ Department of Medicine, Internal Medicine and Rheumatology Unit, Azienda Ospedaliero-Universitaria di Parma, Italy; ${ }^{8}$ Department of Medical Sciences, Rheumatology Section, University of Ferrara, Italy; ${ }^{9}$ Rheumatology Unit, University Hospital, Turin, Italy; ${ }^{10}$ ASST Grande Ospedale Metropolitano Niguarda, Milan, Italy

\begin{abstract}
SUMMARY
Over the last few years, the landscape of treatments for axial spondyloarthritis ( $\mathrm{SpA}$ ) has been rapidly evolving, urging international scientific societies to draft or update existing clinical practice guidelines (CPGs) on the management of axial SpA. The Italian Society for Rheumatology (SIR) committed to provide revised and adapted evidence- and expert-based recommendations for the management of patients with axial SpA in Italy. A systematic approach to the adaptation of existing CPGs - the ADAPTE methodology - was adopted to obtain updated recommendations suitable for the Italian context. A systematic literature search was performed in Medline and Embase databases to find international CPGs and consensus statements with recommendations for the management of axial SpA published in the previous five years. A working group composed of rheumatologists with proven experience in the management of axial SpA and methodologists identified the key research questions which guided study selection and data extraction. Guideline quality was assessed using the Appraisal of Guidelines Research and Evaluation (AGREE) II tool. The Italian recommendations were developed by endorsing or adapting and rewording some existing recommendations. The draft of the recommendations was sent to a multidisciplinary group of external reviewers for comment and rating.

Six original CPGs were selected and used to create this SIR CPG, which includes a final set of 14 recommendations covering the management of patients with axial SpA across the following domains: assessment, pharmacological and non-pharmacological treatment, and follow-up.

The dissemination and implementation of these SIR recommendations are expected to support an evidencebased clinical approach to the management of patients with axial SpA in Italy.
\end{abstract}

Key words: Clinical Practice Guideline, axial spondyloarthritis, management; treatment.

Reumatismo, 2021; 73 (2): 71-88

\section{INTRODUCTION}

The term spondyloarthritis (SpA) encompasses a group of inflammatory disorders which share some typical clinical features: mainly inflammatory back pain due to sacroiliitis and spondylitis, but also enthesitis, peripheral arthritis, dactylitis, and extra-skeletal manifestations such as psoriasis, uveitis, or inflammatory bowel diseases (IBD) (1). A decade ago, the As- sessment of Spondyloarthritis International Society (ASAS) contributed to distinguish patients with predominantly axial symptoms from those in which peripheral arthritis was prevalent, providing classification criteria for axial $\mathrm{SpA}$ and peripheral $\mathrm{SpA}$, respectively $(2,3)$.

According to these criteria, axial SpA comprises Ankylosing Spondylitis (AS), defined by the presence of radiographic sacroiliitis according to the 1984 modified New York $\overline{\text { Corresponding author: }}$ Maria Manara

Division of Rheumatology, ASST Gaetano Pini-CTO Piazza Cardinal Ferrari, 1 20122 Milano (MI), Italy E-mail: maria.manara@gmail.com 
classification criteria, and non-radiographic axial SpA (nr-axial SpA), characterized by axial symptoms and other clinical, laboratory, and imaging features without structural changes in the radiograph of the sacroiliac (SI) joints (4). For the definition of nr-axial SpA, a valuable support is provided by the inflammatory changes in the SI joints that may be revealed by magnetic resonance imaging (MRI) (5).

In the past, the treatment of axial SpA was mainly based on physiotherapy and the use of non-steroidal anti-inflammatory drugs (NSAIDs). The advent of biological disease-modifying anti-rheumatic drugs (bDMARDs) has dramatically improved the outcome of the patients, allowing them to achieve a better control of inflammation and therefore to prevent or delay the progression toward ankylosis (6). Over the last years, the continuous development of new therapies has led national and international scientific societies to draft or update existing guidelines for the management of axial SpA, in order to help rheumatologists to deal with the broad range of different mechanisms of action offered by current treatments.

On this basis, the Italian Society for Rheumatology (SIR) decided to draft national guidelines on the management of patients with axial SpA in 2018. A systematic approach was adopted to adapt existing guidelines - the ADAPTE methodology - and update them to make them suitable for the Italian context $(7,8)$.

\section{Objective}

The aim of this guideline is to provide revised and adapted, evidence- and expertbased recommendations for the management of patients with axial SpA in Italy.

\section{Target patient population}

Adult patients (age $\geq 18$ years) with axial SpA (AS) or nr-axial SpA with a diagnosis formulated by a rheumatologist and guided, when appropriate, by the ASAS classification criteria for axial SpA or other criteria.

\section{Target users}

Rheumatologists and physicians (general practitioners, internists, physiatrists, radiol- ogists) or health professionals who manage patients with axial SpA in primary care and hospital, community and academic practice settings.

\section{What is covered}

These recommendations focus on the management of patients with axial SpA across the following domains: assessment, pharmacological and non-pharmacological treatment, and follow-up.

\section{Areas that are not covered}

The surgical treatment of axial SpA was not included in this guideline.

\section{Funding}

These recommendations were drafted with no specific funding from any bodies in the public, commercial sectors or non-profit organization. Non-economic support, such as meeting rooms and secretariat services, was provided by SIR to carry out the work described in this manuscript.

\section{MATERIALS AND METHODS}

\section{Approach to guideline development}

A systematic approach of guideline adaptation based on the standards of the ADAPTE collaboration (7) was adopted to identify, appraise, summarize, and customize the existing international guidelines on axial $\mathrm{SpA}$ to adapt them to the needs of the Italian healthcare context. The ADAPTE methodology applied by the SIR to develop national guidelines was described elsewhere (8).

\section{Working Group}

The Working Group consisted of 10 rheumatologists on behalf of the SIR. Six rheumatologists (M.M., I.P., A.B, A.A., S.P., C.S.), and a project coordinator (N.U.) from the SIR Study Centre were responsible for CPG methodology and development. Three experts in the management of axial SpA, designated by the SIR (A.C., S.D., A.M.), were included in each phase of the guideline development by participating to web-meetings (April 2019 and October 2019), contributing to e-mail discussions and attending a working group at the $56^{\text {th }}$ SIR National Meeting (November 2019). 


\section{Stakeholder involvement}

The draft of these recommendations was revised and rated by an external multidisciplinary panel of rheumatologists with known experience in the management of axial SpA (13), a gastroenterologist (1), a physiatrist (1), an ophthalmologist (1), a physiotherapist (1), a nurse (1), and a representative of patients' associations (1) on behalf of the SIR. The recommendations were developed without any input from, or cooperation with, any pharmaceutical company.

\section{Defining the scope}

The scope of the revised guideline and a comprehensive list of potential management questions that needed to be addressed was developed a priori by the working group and agreed by consensus. Fourteen research questions were identified (Table I) and guided the systematic literature search.

\section{Inclusion and exclusion criteria}

International clinical practice guidelines and consensus statements with recommendations for management of axial SpA pub- lished in English or Italian between January 1, 2014, and October 1, 2019, were included. When multiple guidelines from the same scientific society were available, the last updated version was included.

Clinical practice guidelines and consensus statements which were non-original i.e. duplicated, or adaptation of previous recommendations) or did not answer to the key research questions were excluded.

\section{Search strategy}

A systematic literature search according to the above-mentioned criteria was checked by the members of the Study Centre and performed by M.M. and I.P. in Medline and Embase databases combining keywords for axial $\mathrm{SpA}$ and for guidelines and consensus statements (Appendix 1). Furthermore, a grey literature search of rheumatology societies, guideline clearinghouses, cross-references and a gate-keeper (Google Scholar) was performed. All search results were screened by 2 independent reviewers (M.M., I.P.), and disagreements were resolved by consensus or by discussion involving the other members of the Study

Table I - Key questions regarding the overall management of patients with axial spondyloarthritis. Fourteen research question guided the systematic review and the drafting of the adapted recommendations.

\begin{tabular}{|c|c|c|}
\hline N. & Text of the health question & N. recommendation \\
\hline \multicolumn{3}{|c|}{ Assessment of axial SpA } \\
\hline 1 & What is the role of classification criteria in the diagnosis of axial SpA? & 1 \\
\hline 2 & What is the role of laboratory tests in the diagnosis of axial SpA? & $\|$ \\
\hline 3 & What is the role of imaging in the diagnosis of axial SpA? & III \\
\hline 4 & How should disease activity and structural damage be monitored in patients with axial SpA? & IV \\
\hline \multicolumn{3}{|c|}{ Treatment of axial SpA } \\
\hline 5 & What should be the target(s) in axial SpA management? & V \\
\hline 6 & What is the pharmacological treatment of patients with axial involvement? & VI \\
\hline 7 & What is the pharmacological treatment of patients with peripheral arthritis? & VII \\
\hline 8 & What is the pharmacological treatment of patients with enthesitis? & VIII \\
\hline 9 & What is the pharmacological treatment of patients with uveitis? & IX \\
\hline 10 & What is the pharmacological treatment of patients with inflammatory bowel disease? & $\mathrm{X}$ \\
\hline 11 & What is the safety of the pharmacological treatment? & $\mathrm{XI}$ \\
\hline 12 & What is the role of the non-pharmacological treatment in patients with axial SpA? & XII \\
\hline \multicolumn{3}{|c|}{ Monitoring of axial $\mathrm{SpA}$} \\
\hline 13 & What is the role of multidisciplinary collaboration in the management of patients with axial SpA? & XIII \\
\hline 14 & How should long-term complications of axial SpA be managed? & XIV \\
\hline
\end{tabular}




\section{APPENDIX 1 - SEARCH STRATEGY}

\section{DATABASE 1: Medline (via Pubmed)}

(/(“Sacroilititis”[MeSH Terms] OR ("spondylitis, ankylosing”[MeSH Terms] OR "Spondylarthropathies"[MeSH Terms])) OR (/(/(/("Sacroilitis”"Text Word] OR "enthesitis"[Text Word]) OR "sacroileitis"[Text Word]) OR "spondyloarthropathy"[Text Word]) OR "spondyloarthropat"[Text Word]) OR "spondyloarthriti”" [Text Word]) OR ((“"spondylitis, ankylosing"[MeSH Terms] OR ("spondylitis"[All Fields] AND "ankylosing"[All Fields])) OR "ankylosing spondylitis"[All Fields]) OR ("ankylosing"[All Fields] AND "spondylitis"[All Fields]))) OR "axial spondyloarthritis"[Text Word])) AND (U(I(/("Practice Guideline"[Publication Type] OR "Practice Guidelines As Topic"[MeSH Terms]) OR "Practice Guideline"[Text Word]) OR "Practice Guidelines"[Text Word]) OR (((/()(“Guideline"[Publication Type] OR "Guidelines As Topic"[MeSH Terms]) OR "Guideline"[Text Word]) OR "Guidelines"[Text Word]) OR "Guideline"[Title]) OR "Guidelines"[Title]) OR "guidance statement"[Title]) OR "guidance statements"[Title]) OR (/(/(“Consensus Development Conference"[Publication Type] OR "consensus development conference, nih"[Publication Type]) OR "Consensus"[MeSH Terms]) OR "Consensus"[Text Word]) OR "consensuses"[Title]) OR "Consensus"[Title])) OR ("Recommendation"[Text Word] OR "Recommendations"[Text Word])) OR ("Best Practice"[Text Word] OR "Best Practices"[Text Word])) OR ((((" "position statement"[Title] OR "position statements"[Title]) OR "practice parameter"[Title]) OR "practice parameters"[Title]) OR "appropriate use criteria"[Title]) OR "appropriateness criteria"[Title]))) AND “2014/01/01 00:00":"2019/10/01 05:00"[Date - Publication]

\section{DATABASE 2: Embase - no Medline}

'ankylosing spondylitis'/exp OR 'spondylarthritis'/exp OR 'axial spondyloarthritis' OR 'spondyloarthritis' OR 'ankylating spondylitis' OR 'ankylopoietic spondylarthritis' OR 'ankylopoietic spondylitis' OR 'ankylosing spondilitis' OR 'ankylosing spondylarthritis' OR 'ankylotic spondylitis' OR 'Bechterew disease' OR 'spondylarthritis ankylopoietica' OR 'spondylarthritis ankylosans' OR 'spondylarthrosis ankylopoietica' OR 'spondylitis ankylopoetica' OR 'spondylitis ankylopoietica' OR 'spondylitis, ankylosing' OR 'spondyloarthritis ankylopoietica' OR 'vertebral ankylosis' OR 'sacroilitis'/exp OR 'sacroilitis' OR 'enthesitis'/exp OR 'enthesitis' AND

('practice guideline'/exp OR 'practice guideline' OR 'practice guidelines'/exp OR 'practice guidelines' OR 'clinical practice guideline'/exp OR 'clinical practice guideline' OR 'clinical practice guidelines'/exp OR 'clinical practice guidelines' OR 'clinical practice guidelines as topic'/ exp OR 'clinical practice guidelines as topic' OR 'good clinical practice'/exp OR 'good clinical practice' OR 'guideline'/exp OR 'guideline' OR 'guidelines'/exp OR 'guidelines' OR 'guidelines as topic'/exp OR 'guidelines as topic' OR 'health planning guidelines' OR 'health planning guideline' OR 'treatment guidelines'/exp OR 'clinical pathway'/exp OR 'clinical pathway' OR 'clinical pathways' OR 'consensus development'/ exp OR 'consensus development' OR 'consensus development conference'/exp OR 'consensus development conference' OR 'consensus development conferences'/exp OR 'consensus development conferences' OR 'consensus development conferences as topic'/exp OR 'consensus development conferences as topic' OR 'consensus development conference, NIH' OR 'consensus'/exp OR 'consensus' OR 'consensuses' OR recommendat* OR 'position statement' OR 'position statements' OR 'policy statement' OR 'policy statements' OR 'practice parameter' OR 'practice parameters' OR 'best practice' OR 'best practices' OR 'standards' OR CPG*)

AND [2014-2019]/py AND [embase]/lim NOT [medline]/lim

Centre. A flow chart of search results is shown in Figure 1.

\section{Appraisal of guideline quality}

Guideline quality was assessed by two raters (M.M., I.P.) using the on-line Appraisal of Guidelines Research and Evaluation (AGREE) II instrument (9). The final AGREE score was not considered as a criterion for exclusion.

\section{Level of evidence and strength of recommendation}

Different grading systems for evidence were used across guidelines. In order to reconcile these differences, the grading system of each guideline was revised and reported by rating the level of evidence and strength of each recommendation in accordance with the Oxford Levels of Evidence (10) (Table II). In case of disagreement, the rating of each recommendation based on the most updated evidence was considered.

\section{Evidence framework and development of recommendations}

For each key question, evidence tables containing guideline characteristics, recommendations, AGREE summary scores, and level of evidence and strength of recommendation according to the original grading system were prepared. An Italian recommendation was developed by endorsement or adaption and rewording of existing recommendations. This guidance was prepared in accordance with the AGREE reporting checklist (11). 


\section{Peer review}

The draft of the recommendations developed by the working group was sent to external reviewers $(n=25)$ for comment and rating. An online survey was performed via Google Forms between May $22^{\text {nd }} 2020$ and June $22^{\text {nd }} 2020$ and feedback from respondents $(n=19$, response rate $76 \%)$ was considered to finalize these recommendations. The final document underwent a peer review process through the journal Reumatismo.

\section{RESULTS}

\section{Key to understanding this guidance}

Each recommendation is presented with a level of evidence and strength and is accompanied by a supporting text that is structured as follows:

Summary of guidelines. A summary of recommendations made by the axial SpA guidelines identified in the systematic review.

Recommendation/supporting evidence. Specific source guidelines that were used for adaptation.

Evidence to recommendation. Discussion of the guideline panel about the adapted recommendation with regards to further specifications and comments on the sources used to develop the recommendation.

The level of agreement of external reviewers for each recommendation is reported in Table III.

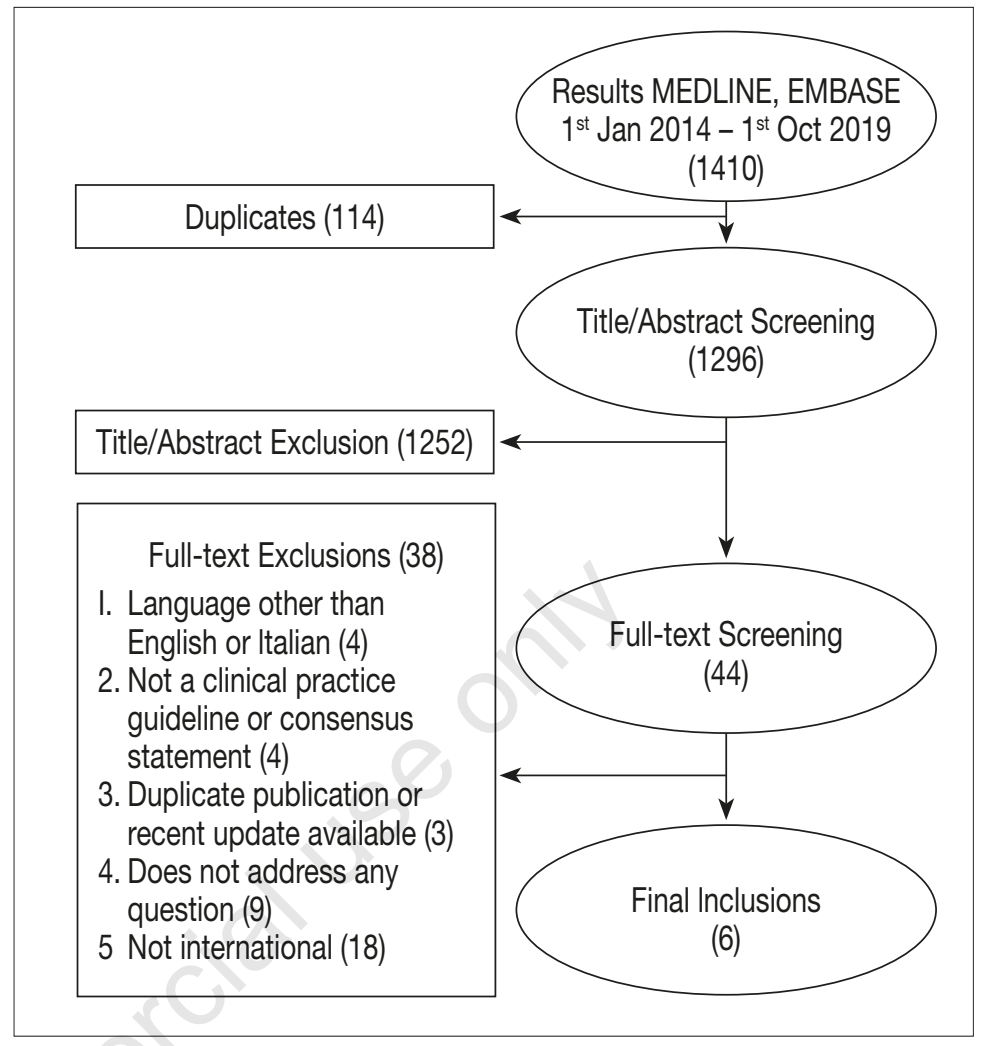

Figure 1 - Steps in the systematic review of guidelines on diagnosis and treatment of axial spondyloarthritis.

\section{Recommendations}

Six original clinical practice guidelines (CPGs) (12-17) were selected and used to accomplish this SIR CPG made of 14 recommendations (Table III).

Table II - Guidance to categories of evidence and strength of recommendations based on the Oxford Levels of Evidence (10).

\begin{tabular}{|c|l|}
\hline Category & Evidence \\
\hline 1 & From meta-analysis of randomised controlled trials or from at least one randomised controlled trial \\
\hline 2 & From at least one controlled study without randomisation or from at least one cohort study \\
\hline 3 & From at least one case-control study \\
\hline 4 & From case-series or poor-quality cohort and case-control studies \\
\hline 5 & From expert committee reports or opinions and/or clinical experience of respected authorities \\
\hline Grade & Strength \\
\hline A & consistent level 1 studies \\
\hline B & consistent level 2 or 3 studies or extrapolations ${ }^{\star}$ from level 1 studies \\
\hline C & level 4 studies or extrapolations ${ }^{\star}$ from level 2 or 3 studies \\
\hline D & level 5 evidence or troublingly inconsistent or inconclusive studies of any level \\
\hline
\end{tabular}

${ }^{*}$ Extrapolations are where data is used in a situation with potential clinically important differences compared to the original study situation. 
Table III - The final set of 14 recommendations for the management of axial spondyloarthritis.

\begin{tabular}{|c|c|c|c|c|}
\hline & Recommendations & LoE & SoR & $\begin{array}{l}\text { LoA, } \\
\text { median (IQR) }\end{array}$ \\
\hline 1 & $\begin{array}{l}\text { In specialist care settings, diagnosis of axial SpA should be made by a rheumatologist and } \\
\text { based on clinical features and imaging and laboratory findings. }\end{array}$ & 5 & D & $10(9,10)$ \\
\hline 2 & $\begin{array}{l}\text { Do not rule out a diagnosis of axial SpA solely on the basis of a negative HLA-B27 result or of a } \\
\text { normal C-reactive protein (CRP) and erythrocyte sedimentation rate (ESR). }\end{array}$ & 5 & D & $10(8,10)$ \\
\hline 3 & $\begin{array}{l}\text { In general, conventional radiography of the SI joints is recommended as the first imaging } \\
\text { method to detect sacroilitis as part of axial SpA. In certain cases, such as in young patients } \\
\text { and those with short symptom duration, MRI of the SI joints is an alternative first imaging } \\
\text { method. } \\
\text { If the diagnosis of axial SpA cannot be established based on clinical features and } \\
\text { conventional radiography, and axial SpA is still suspected, MRI of the SI joints is } \\
\text { recommended. } \\
\text { Use the ASAS MRI criteria to interpret MRI of SI joints. If the MRI meets the ASAS MRI } \\
\text { criteria, nr-axial SpA is very likely. } \\
\text { MRI of the spine is not generally recommended to diagnose axial SpA. } \\
\text { Imaging modalities, other than conventional radiography and MRI are generally not } \\
\text { recommended in the diagnosis of axial SpA. }\end{array}$ & 3 & $B$ & $9(7,10)$ \\
\hline 4 & $\begin{array}{l}\text { Disease monitoring of patients with axial SpA should include patient-reported outcomes, } \\
\text { clinical findings, laboratory tests and imaging, all with the appropriate instruments and } \\
\text { relevant to the clinical presentation the clinical presentation. The frequency of monitoring } \\
\text { should be decided on an individual basis depending on symptoms, severity and treatment. } \\
\text { MRI of the SI joints and/or the spine may be used to assess and monitor disease activity, } \\
\text { while conventional radiography of the SI joints and/or spine may be used for long-term } \\
\text { monitoring of structural damage, particularly new bone formation. }\end{array}$ & 5 & B & $9(8,10)$ \\
\hline 5 & $\begin{array}{l}\text { Treatment should be guided according to a predefined target, which must be based on a } \\
\text { shared decision between the patient and the rheumatologist. } \\
\text { The treatment target should be clinical remission/inactive disease of musculoskeletal and } \\
\text { extra-articular manifestations and should be individualized based on the current clinical } \\
\text { manifestations of the disease. Once the target is achieved, it should ideally be maintained } \\
\text { throughout the course of the disease. } \\
\text { Validated measures of musculoskeletal disease activity may be used in clinical practice } \\
\text { to support treatment decisions based on the physician assessment; the frequency of the } \\
\text { measurements depends on the level of disease activity. In addition to clinical and laboratory } \\
\text { measures, imaging results may be considered in clinical management. }\end{array}$ & 5 & $D$ & $9(7,10)$ \\
\hline 6 & $\begin{array}{l}\text { In patients with axial SpA and predominant axial involvement, as first-line pharmacological } \\
\text { treatment offer NSAIDs at the lowest effective dose. If an NSAID taken at the maximum } \\
\text { tolerated dose for } 2 \text { weeks does not provide adequate pain relief, consider switching to } \\
\text { another NSAID. For patients who respond well to NSAIDs, continuous use is preferred to on- } \\
\text { demand use. } \\
\text { Patients with axial disease should not receive treatment with systemic glucocorticoids. } \\
\text { Glucocorticoid injections directed to the local site of musculoskeletal inflammation may be } \\
\text { considered in case of isolated active sacroiliitis, despite treatment with NSAIDs. } \\
\text { Patients with purely axial disease should not be treated with csDMARDs. } \\
\text { In patients with persistently high disease activity despite treatment with NSAIDs, bDMARDs } \\
\text { should be considered. } \\
\text { As first line bDMARD, either TNFi or IL-17i may be used. The choice of treatment should } \\
\text { consider associated conditions such as extra articular manifestations. } \\
\text { In patients with primary non response to a first bDMARD, treatment with a different class } \\
\text { of bDMARDs (TNFi or IL-17i) may be preferred to the use of a molecule with the same } \\
\text { mechanism of action. In patients with secondary non response to a first bDMARD, treatment } \\
\text { with a different molecule with the same mechanism of action may be considered. } \\
\text { In patients with stable axial disease on bDMARD, on-demand treatment with NSAIDs may be } \\
\text { preferred to continuous treatment with NSAIDs. In those receiving treatment with a bDMARD and } \\
\text { NSAIDs, continuing treatment with bDMARD alone may be preferred to continuing both treatments. } \\
\text { In patients receiving treatment with a bDMARD, discontinuation of the bDMARD may not be } \\
\text { recommended, while tapering of the bDMARD dose may be considered. }\end{array}$ & $\begin{array}{l}1 \\
5 \\
1 \\
1 \\
5 \\
5\end{array}$ & $\begin{array}{l}\text { B } \\
\text { D } \\
\text { B } \\
\text { A } \\
\text { D } \\
\text { D }\end{array}$ & $9(8,10)$ \\
\hline
\end{tabular}




\begin{tabular}{|c|c|c|c|c|}
\hline & Recommendations & LoE & SoR & $\begin{array}{l}\text { LoA, } \\
\text { median (IQR) }\end{array}$ \\
\hline 7 & $\begin{array}{l}\text { In patients with stable axial disease and active peripheral arthritis despite treatment with NSAIDs, a } \\
\text { treatment with locally administered glucocorticoids may be used. } \\
\text { Sulfasalazine* or methotrexate§ should be considered in patients with stable axial disease and } \\
\text { prominent peripheral arthritis despite treatment with NSAIDs and locally administered glucocorticoids. }\end{array}$ & $\begin{array}{c}5 \\
1^{*} ; 2 \S\end{array}$ & $A^{*} ; B \S$ & $9(7,10)$ \\
\hline 8 & $\begin{array}{l}\text { In patients with stable axial disease and active enthesitis despite treatment with NSAIDs, a } \\
\text { treatment with locally administered glucocorticoids may be used. Peri-tendon injections of } \\
\text { Achilles, patellar, and quadriceps tendons should be avoided. }\end{array}$ & 5 & $\mathrm{D}$ & $9(8,10)$ \\
\hline 9 & $\begin{array}{l}\text { In patients with axial } \mathrm{SpA} \text { and acute uveitis, we recommend multidisciplinary management with an } \\
\text { ophthalmologist to decrease the severity, duration, or complications of episodes. } \\
\text { In these patients, treatment with TNFi monoclonal antibodies may be preferred to treatment with } \\
\text { other biologics. }\end{array}$ & 5 & D & $10(10,10)$ \\
\hline 10 & $\begin{array}{l}\text { In patients with axial } \mathrm{SpA} \text { and active IBD, we recommend referral to a gastroenterologist for the } \\
\text { treatment of IBD. } \\
\text { In patients with axial SpA and inactive IBD, we do not recommend any particular NSAID as the } \\
\text { preferred choice to decrease the risk of worsening of IBD symptoms. } \\
\text { We conditionally recommend treatment with TNFi monoclonal antibodies over treatment with } \\
\text { other biologics. }\end{array}$ & $\begin{array}{l}5 \\
3\end{array}$ & $\begin{array}{l}\mathrm{D} \\
\mathrm{D} \\
\mathrm{C}\end{array}$ & $10(9,10)$ \\
\hline 11 & $\begin{array}{l}\text { Take into account the adverse effects associated with NSAIDs, csDMARDs and bDMARDs, when } \\
\text { monitoring SpA in primary care. } \\
\text { Prior to starting bDMARD, screening for TB, HBV and HCV is recommended. } \\
\text { Treatment for latent TB* and pre-emptive therapy for chronic hepatitis B infection } \S \text { are also } \\
\text { recommended according to local guidelines. } \\
\text { For patients with axial SpA in whom disease cannot otherwise be controlled, TNF inhibitors may } \\
\text { be continued throughout pregnancy. } \\
\text { We strongly recommend checking the vaccination status of patients with axial SpA, following local } \\
\text { guidelines. }\end{array}$ & $\begin{array}{c}5 \\
2 \\
2^{*} ; 5 \S \\
5 \\
5\end{array}$ & $\begin{array}{c}D \\
C \\
C^{*} ; D \S \\
D \\
D\end{array}$ & $9(8,10)$ \\
\hline 12 & $\begin{array}{l}\text { Patients with axial SpA should be referred to a rehabilitation specialist to start an individualized, } \\
\text { structured exercise program. Treatment with physical therapy is recommended in both patients } \\
\text { with active and stable axial SpA. } \\
\text { In patients with spinal fusion or advanced spinal osteoporosis, treatment with spinal manipulation } \\
\text { is not recommended. }\end{array}$ & 1 & $A$ & $10(8,10)$ \\
\hline 13 & $\begin{array}{l}\text { In patients with axial SpA, the management of musculoskeletal and extra-articular manifestations } \\
\text { should be coordinated, as needed, between the rheumatologist and other specialists. }\end{array}$ & 5 & $D$ & $10(9,10)$ \\
\hline 14 & $\begin{array}{l}\text { Discuss risk factors for cardiovascular comorbidities with all people with axial SpA. } \\
\text { Consider regular osteoporosis assessments for people with axial SpA. Advise people with axial } \\
\text { SpA that they may be prone to fractures and should consult a healthcare professional following } \\
\text { falls or physical trauma, particularly in the event of increased musculoskeletal pain. }\end{array}$ & $\begin{array}{l}5 \\
5\end{array}$ & $\begin{array}{l}\text { D } \\
D\end{array}$ & $10(8,10)$ \\
\hline
\end{tabular}

LoE, level of evidence; SoR, strength of the recommendation; LoA, level of agreement of the stakeholders; IQR, interquartile range; SpA, spondyloarthritis; SI, sacroiliac; MRI, magnetic resonance; ASAS, Assessment of Spondyloarthritis International Society; NSAID, non-steroidal anti-inflammatory drug; csDMARDs, conventional synthetic disease-modifying anti-rheumatic drugs; bDMARDs, biological disease-modifying anti-rheumatic drugs; TNFi, tumor necrosis factor inhibitors; IL-17i, IL-17 inhibitors; IBD, inflammatory bowel disease; TB, tuberculosis; HBV, hepatitis B virus; HCV, hepatitis C virus.

\section{Recommendations for assessment of axial SpA}

\section{RECOMMENDATION 1 Clinical criteria}

In specialist care settings, diagnosis of axial SpA should be made by a rheumatologist and based on clinical features and imaging and laboratory findings (level 5; Strength D).
Summary of guidelines. The search identified only 1 CPG that addressed the clinical assessment of axial SpA (15). This CPG suggests using validated SpA criteria to guide clinical judgement when diagnosing SpA.

Recommendation/supporting evidence. NICE (15).

Evidence to recommendation. The panel did not agree upon the endorsement of the NICE recommendation and underlined that classification criteria are not intended for 
diagnostic purposes, even if they can help in the diagnosis (18). A knowledgeable rheumatologist should make a diagnosis based on the full evaluation of all clinical, laboratory and imaging information, and the opinion of an expert rheumatologist remains the reference standard for diagnosis. Examples of classification criteria include Amor and European Spondyloarthropathy Study Group (ESSG) criteria (19) for general SpA criteria and ASAS (axial) (2), Rome (20) and modified New York criteria (4) for axial SpA criteria.

According to ASAS criteria for axial SpA, a patient with chronic low back pain $(\geq 3$ months) and age at onset less than 45 years can be classified as having axial SpA in the presence of sacroiliitis in standard radiography or MRI plus at least one typical SpA feature, or in the presence of HLA-B27 plus at least two other SpA features. Typical SpA features include: inflammatory back pain, arthritis, enthesitis (especially of the heel), uveitis, dactylitis, psoriasis, inflammatory bowel disease, good response to NSAIDs, family history of SpA, HLAB27, elevated CRP (2).

\section{RECOMMENDATION 2}

\section{Laboratory tests}

Do not rule out a diagnosis of axial SpA solely on the basis of a negative HLAB27 result or of a normal C-reactive protein (CRP) and erythrocyte sedimentation rate (ESR) (level 5; Strength D).

Summary of guidelines. The search identified 1 CPG that addressed the role of laboratory tests in the assessment of axial SpA (15).

Recommendation/supporting evidence. NICE (15).

Evidence to recommendation. The panel agreed upon the complementary role of laboratory investigations (HLA-B27, acute phase reactants) in the diagnosis of axial $\mathrm{SpA}$, which is mainly based on clinical criteria.

\section{RECOMMENDATION 3 Imaging}

In general, conventional radiography of the SI joints is recommended as the first imaging method to detect sacroiliitis as part of axial SpA. In certain cases, such as in young patients and those with short symptom duration, MRI of the SI joints is an alternative first imaging method.

If the diagnosis of axial SpA cannot be established based on clinical features and conventional radiography, and axial $\mathrm{SpA}$ is still suspected, MRI of the SI joints is recommended.

Use the ASAS MRI criteria to interpret MRI of SI joints. If the MRI meets the ASAS MRI criteria, nr-axial SpA is very likely.

MRI of the spine is not generally recommended to diagnose axial SpA.

Imaging modalities, other than conventional radiography and MRI are generally not recommended in the diagnosis of axial SpA (level 3; Strength B).

Summary of guidelines. The search identified 2 CPGs that addressed imaging modalities for the assessment of axial SpA $(14,15)$. Both CPGs recommend conventional radiography of the SI joints as the first imaging method to detect sacroiliitis in axial SpA $(14,15)$. MRI of the SI joints is recommended when the diagnosis of axial $\mathrm{SpA}$ cannot be established based on clinical features and conventional radiography $(14,15)$. Both CPGs do not recommend the use of MRI of the spine, as first technique, for the diagnosis of axial SpA, as well as other imaging modalities such as scintigraphy $(14,15)$.

Recommendation/supporting evidence. NICE, EULAR imaging $(14,15)$.

Evidence to recommendation. The panel agreed to recommend conventional radiography of the SI joints as the first imaging method for the diagnosis of axial SpA. If a plain radiograph shows sacroiliitis meeting the modified New York criteria (bilateral grade 2-4 or unilateral grade 3-4 sacroiliitis), diagnosis of axial SpA (AS) can be made (4). When the plain radiograph does not show sacroiliitis fulfilling modified New York criteria, or a radiograph is not appropriate, MRI of the SI joints should be requested.

In case of absence of radiographic sacro- 
iliitis, the diagnosis of axial SpA can be made based on the MRI of the SI joints, according to the ASAS MRI criteria $(5,21)$. If MRI does not meet the criteria, the possibility of axial SpA cannot be excluded, and therefore an HLA-B27 test should be offered, if not yet performed. If it is positive, the diagnosis should be based on clinical features. If a diagnosis of axial SpA cannot be confirmed and clinical suspicion remains high, a follow-up MRI should be considered (15).

The panel agreed that the MRI of the spine is not generally recommended to diagnose axial SpA. However, in certain cases its prescription may be indicated, especially when MRI of the SI joints does not allow a definite diagnosis and the patient symptoms are suggestive of a spinal involvement, as recommended by some recent national CPGs and expert consensus $(22,23)$. Scintigraphy and other imaging modalities are not recommended for the diagnosis of axial SpA, even if computed tomography (CT) may provide additional information on structural damage, when conventional radiography is negative and MRI cannot be performed (14). Ultrasound (US) and MRI of enthesis may support the diagnosis of axial $\mathrm{SpA}$ in patients with peripheral enthesitis.

\section{RECOMMENDATION 4}

\section{Disease activity assessment}

Disease monitoring of patients with axial $\mathrm{SpA}$ should include patient-reported outcomes, clinical findings, laboratory tests and imaging, all with the appropriate instruments and relevant to the clinical presentation. The freqeuncy of monitoring should be decided on an individual basis depending on symptoms, severity and treatment (level 5; Strength D).

The MRI of the SI joints and/or the spine may be used to assess and monitor disease activity, while conventional radiography of the SI joints and/or spine may be used for long-term monitoring of structural damage, particularly new bone formation (level 2; Strength B).
Summary of guidelines. The search identified 3 CPGs that addressed disease monitoring of axial SpA (12-14). Disease monitoring with clinical, laboratory and imaging findings is recommended for all patients with axial SpA (13). The ACR CPGs recommend to perform disease activity measurements and laboratory assessment (ESR and CRP) periodically, but discourage the use of repeated imaging at scheduled intervals as a standard approach (12). MRI may be helpful to assess disease activity, while conventional radiography may be used for long-term monitoring of structural damage (14).

Recommendation/supporting evidence. ASAS-EULAR 2016; EULAR imaging 2015; ACR 2019 (12-14).

Evidence to recommendation. The panel agreed to recommend disease monitoring through clinical findings, laboratory tests and imaging. In adults with active or stable axial SpA, periodic monitoring of CRP concentrations or ESR and of validated disease activity measures (ASDAS, BASDAI) may be recommended. Imaging modalities should be individualized on each patient. When monitoring disease activity, MRI with STIR sequences is indicated to detect inflammation, while conventional radiography may be used for long-term monitoring of structural damage, particularly new bone formation.

\section{Recommendations for treatment of axial SpA}

\section{RECOMMENDATION 5 Treatment target}

Treatment should be guided according to a predefined target, which must be based on a shared decision between patient and rheumatologist.

The treatment target should be clinical remission/inactive disease of musculoskeletal and extra-articular manifestations and should be individualized based on the current clinical manifestations of the disease. Once the target is achieved, it should ideally be maintained throughout the course of the disease. 
Validated measures of musculoskeletal disease activity may be used in clinical practice to support treatment decisions based on physician assessment; the frequency of the measurements depends on the level of disease activity. In addition to clinical and laboratory measures, imaging results may be considered in clinical management (level 5; Strength D).

Summary of guidelines. The search identified 3 CPGs that addressed the treatment target in the management of axial $\operatorname{SpA}(12,13$, 16). Treatment should be guided on the basis of a predefined target, which should be individualized for every patient $(13,16)$. While one CPG is entirely focused on the implementation of treat-to-target strategies (16), the ACR 2019 recommendations are more in favor of a strategy based on physician assessment than a treat-to-target one (12).

Recommendation/supporting evidence. ASAS-EULAR 2016; SMOLEN 2017; ACR 2019 (12, 13, 16).

Evidence to recommendation. The panel acknowledged the importance of a treatment target aimed at remission or disease inactivity. In the absence of clear evidence on the efficacy of a treat-to-target strategy based on clinimetric indexes in axial SpA, the panel agreed to modify the original statement of the 'treat-to-target' recommendations by Smolen and colleagues (16), to underline that disease activity measures may support treatment decisions, which should be mainly based on clinical assessment.

\section{RECOMMENDATION 6 Treatment of patients with axial involvement}

In patients with axial SpA and predominant axial involvement, as first-line pharmacological treatment offer NSAIDs at the lowest effective dose. If an NSAID taken at the maximum tolerated dose for 2 weeks does not provide adequate pain relief, consider switching to another NSAID. For patients who respond well to NSAIDs, continuous use is preferred to on-demand use (level 1; Strength A).

Patients with axial disease should not re- ceive treatment with systemic glucocorticoids (level 1; Strength B).

Glucorticoid injections directed to the local site of musculoskeletal inflammation may be considered in case of isolated active sacroiliitis, despite treatment with NSAIDs (level 5; Strength D).

Patients with purely axial disease should not be treated with conventional synthetic disease-modifying anti-rheumatic drugs (csDMARDs) (level 1; Strength B).

In patients with persistently high disease activity despite treatment with NSAIDs, bDMARDs should be considered (level 1; Strength A).

As first line bDMARD, either TNF $\alpha$ inhibitors (TNFi) or IL-17 inhibitors (IL17i) may be used. The choice of treatment should consider associated conditions such as extra-articular manifestations (level 5; Strength D).

In patients with primary non response to a first bDMARD, treatment with a different class of bDMARDs (TNFi or IL-17i) may be preferred to the use of a molecule with the same mechanism of action. In patients with secondary non response to a first bDMARD, treatment with a different molecule with the same mechanism of action may be considered (level 5; Strength D).

In patients with stable axial disease on bDMARD, on-demand treatment with NSAIDs may be preferred to continuous treatment with NSAIDs. In those receiving treatment with a bDMARD and NSAIDs, continuing treatment with bDMARD alone may be preferred to continuing both treatments (level 1; Strength B).

In patients receiving treatment with a bDMARD, discontinuation of the bDMARD may not be recommended, while tapering of the bDMARD dose may be considered (level 1; Strength B).

Summary of guidelines. The search identified 4 CPGs that addressed the pharmacological treatment of patients with axial involvement $(12,13,15,17)$. NSAIDs are considered the first-line therapy in all CPGs $(12,13,15,17)$. The ASAS-EULAR CPG 
also mentions analgesic use (13), while systemic glucocorticoids use is not recommended by 3 CPGs $(12,13,17)$. The treatment with csDMARDs should not be used for purely axial disease according to 2 CPGs $(12,13)$, while it is conditionally recommended by APLAR CPG in resourcepoor settings (17).

The treatment with bDMARDs is indicated in patients with active disease, despite NSAID treatment $(12,13,15,17)$. Two CPGs recommend TNFi as first bDMARD $(15,17)$; ASAS-EULAR CPG states that 'current practice is to start with TNFi therapy' (13); the ACR CPG conditionally recommends treatment with TNFi over treatment with IL-17i (12).

In patients who failed a treatment with a first TNFi, three CPGs recommend switching to another TNFi or to an IL-17i (13, 15, 17). The ACR CPG differentiates between primary non responders to $\mathrm{TNFi}$, for whom treatment with an IL-17i should be preferred to treatment with another TNFi, and secondary non responders, for whom treatment with another TNFi should be preferred to treatment with an IL-17i (12).

In patients with stable disease, continuation of the bDMARD is recommended, while NSAID should be discontinued or used on-demand (12). Tapering of bDMARDs is accepted by two CPGs $(13,17)$, but it is not suggested as a standard approach by the last ACR CPG (12).

Recommendation/supporting evidence. NICE; ASAS-EULAR 2016; APLAR 2018; ACR 2019 (12, 13, 15, 17).

Evidence to recommendation. The panel agreed upon the use of NSAIDs as first-line therapy, and underlines the importance of appropriate clinical assessment, ongoing monitoring of risk factors, and the use of a gastroprotective treatment, when suitable. The panel suggested continuous use over on demand use for all NSAIDs, even if the higher efficacy of continuous use in slowing radiographic progression was demonstrated only in a trial with patients treated mainly with celecoxib, while a more recent trial on diclofenac failed to demonstrate a similar effect $(24,25)$. The panel decided not to include a specific recommendation on analgesics use, since the treatment with this pharmacological class is not supported by evidence in axial $\mathrm{SpA}$. According to a more recent ACR CPG, the treatment with systemic glucocorticoids is not recommended, but only local injection is accepted in case of isolated sacroiliitis (12).

The panel suggests that in patients with active axial disease, despite treatment with NSAIDs, sulfasalazine may be considered only when a treatment with bDMARDs is not feasible, based on the results of a recent RCT which showed a beneficial effect of sulfalazine on axial symptoms (26), despite inconclusive results of other previous trials (12). The treatment with methotrexate is not supported by evidence in axial SpA. Even if a dose ranging study on tofacitinib provided some initial evidence about its efficacy on axial symptoms (27), this treatment cannot be recommended at the moment.

The panel agreed upon the use of bDMARDs in patients with active disease, despite treatment with NSAIDs, i.e. patients with an ASDAS $\geq 2.1$ or BASDAI $\geq 4$ and a positive opinion of the rheumatologist to start a treatment with bDMARDs (13). The panel decided not to recommend one mechanism of action over another, since there is no head-to-head comparison between TNFi and IL-17i showing a better performance of one of these treatments in bio-naïve patients with axial SpA. The choice of the treatment should be guided by the presence of comorbidities and associated manifestations. Attention should be paid to disease phenotype (AS or nr-ax SpA), since not all TNFi and IL-17i are licensed for both conditions. In patients receiving treatment with bDMARDs, co-treatment with low-dose MTX is not suggested, since there is no evidence of a better efficacy for the combination therapy in axial SpA (12).

The response to treatment with bDMARDs (TNF $\alpha$ and IL-17 inhibitors) should be assessed 12 weeks after the start of treatment. Treatment should be continued if there is clear evidence of response, defined as an improvement in ASDAS $\geq 1.1$ or an improvement in the Bath Ankylosing Spondylitis Disease Activity Index (BASDAI) 
score $\geq 2(0-10)$ and a positive opinion of the rheumatologist to continue (13).

In patients with active disease, despite the use of the first bDMARD, the panel adopted the distinction made by the last ACR CPG between patients with a primary non response and those with a secondary non response (i.e. patients who relapsed after an initial response to the treatment). A switch toward a different mechanism of action is suggested in patients with a primary non response, while the introduction of another drug with the same mechanism of action may be considered in patients with a secondary non response.

The panel underlined that treatment costs should be considered among other factors, when choosing a bDMARD, always in the best interest of the patient. Currently, the use of biosimilars is suggested in Italy for patients naïve to that class of bDMARDS, while in patients already receiving treatment with an originator bDMARD, switching to its biosimilar is not recommended, although it may be conditional to the NHS policy (28).

The panel agreed not to recommend bDMARD discontinuation in patients with stable disease, but suggested that tapering (i.e. dose reduction or progressive spacing of drug administrations) may be considered in some patients.

\section{RECOMMENDATION 7}

\section{Treatment of patients with peripheral arthritis}

In patients with stable axial disease and active peripheral arthritis despite treatment with NSAIDs, a treatment with locally administered glucocorticoids may be used (level 5; Strength D).

Sulfasalazine* or methotrexate§ should be considered in patients with stable axial disease and prominent peripheral arthritis despite treatment with NSAIDs and locally administered glucocorticoids (level $1 *$ - $2 \S$; Strength A*- B§).

Summary of guidelines. The search identified 4 CPGs that addressed the treatment of peripheral arthritis $(12,13,15,17)$. A treatment with local glucocorticoids is suggest- ed by 2 of them $(12,15)$, while csDMARDs are indicated by all guidelines, in particular sulfasalazine by ASAS-EULAR CPG (13) and sulfasalazine and methotrexate by ACR CPG (12).

Recommendation/supporting evidence. NICE; ASAS-EULAR 2016; ACR 2019; APLAR 2018 (12, 13, 15, 17).

Evidence to recommendation. The panel agreed upon the use of local glucocorticoids in patients with stable axial disease and active peripheral arthritis, despite NSAIDs. If a local treatment does not lead to an adequate control of peripheral arthritis, csDMARDs (sulfasalazine or methotrexate) should be considered.

\section{RECOMMENDATION 8 Treatment of patients with enthesitis}

In patients with stable axial disease and active enthesitis despite treatment with NSAIDs, a treatment with locally administered glucocorticoids may be used. Peri-tendon injections of Achilles, patellar, and quadriceps tendons should be avoided (level 5; Strength D).

Summary of guidelines. The search identified $1 \mathrm{CPG}$ that addressed the treatment of enthesitis, suggesting the use of locally administered glucocorticoids (12).

Recommendation/supporting evidence. ACR 2019 (12).

Evidence to recommendation. The panel agreed upon the use of locally administered glucocorticoids in patients with active enthesitis despite treatment with NSAIDs and a stable axial disease. This treatment is not indicated in specific anatomic region as Achilles, patellar, and quadriceps tendons, due to an increased risk of tendon rupture.

\section{RECOMMENDATION 9}

\section{Treatment of patients with uveitis}

In patients with axial $\mathrm{SpA}$ and acute uveitis, we recommend multidisciplinary management with an ophthalmologist to decrease the severity, duration, or complications of episodes (level 5; Strength D).

In these patients, the treatment with TNFi 
monoclonal antibodies may be preferred to the treatment with other biologics (level 3; Strength C).

Summary of guidelines. The search identified 2 CPGs that addressed the treatment of patients with uveitis $(12,17)$. One CPG suggested referral to an ophthalmologist for the treatment of uveitis (12). The same CPG suggested prescription of topical glucocorticoids for at-home use in the management of symptomatic uveitis (12). Two CPGs recommended use of TNFi monoclonal antibodies over treatment with other biologics $(12,17)$.

Recommendation/supporting evidence. ACR 2019, APLAR $2018(12,17)$.

Evidence to recommendation. The panel agreed upon the importance of specialist referral for the management of uveitis, but it doesn't recommend ophthalmological screening in asymptomatic patients. The panel decided not to suggest the use of self-prescribed medications (topical glucocorticoids) for the management of symptomatic uveitis (recommendation which was based on a very low evidence in the original CPG), underlying the need for referral to the specialist for drug prescription. Based on data from observational studies, the rates of uveitis occurrence seemed lower in patients treated with infliximab and adalimumab compared to etanercept (29); and secukinumab did not seem to be efficacious in the treatment of uveitis (30). Therefore, the panel stated that the treatment with TNFi monoclonal antibodies may be preferred to other mechanisms of action in these patients.

\section{RECOMMENDATION 10}

\section{Treatment of patients with IBD}

In patients with axial $\mathrm{SpA}$ and active IBD, we recommend referral to a gastroenterologist for the treatment of IBD (level 5; Strength D).

In patients with axial $\mathrm{SpA}$ and inactive IBD, we do not recommend any particular NSAID as the preferred choice to decrease the risk of worsening of IBD symptoms (level 5; Strength D).

We conditionally recommend treatment with TNFi monoclonal antibodies over treatment with other biologics (level 3; Strength C).

Summary of guidelines. The search identified 2 CPGs that addressed the treatment of patients with concomitant IBD $(12,17)$. The ACR CPG does not recommend any particular NSAID for the management of musculoskeletal symptoms in patients with inactive IBD (12). Treatment with TNFi monoclonal antibodies is recommended over other mechanisms of action $(12,17)$. Recommendation/supporting evidence. ACR 2019, APLAR $2018(12,17)$.

Evidence to recommendation. The panel stated to highlight the need of specialistic referral for the management of IBD as first recommendation, thus pointing out the importance of multidisciplinary collaboration in the management of patients with axial SpA and IBD. Gastroenterological and/or endoscopy screening is not recommended in asymptomatic patients. The panel agreed upon the absence of a preferred NSAID in the management of associated musculoskeletal symptoms in patients with inactive IBD. Treatment with TNFi monoclonal antibodies was recommended over other mechanisms of action based on evidence of a lower risk of IBD flares in patients treated with infliximab and adalimumab compared to etanercept $(12,31)$ and of an increased risk of IBD exacerbation with IL-17i (32, 33). Therefore, the panel agreed not to recommend etanercept and IL17i in patients with axial $\mathrm{SpA}$ and IBD.

\section{RECOMMENDATION 11 Safety}

Take into account the adverse effects associated with NSAIDs, csDMARDs and bDMARDs, when monitoring SpA in primary care (level 5; Strength D).

Prior to starting a bDMARD, screening for tuberculosis (TB), Hepatitis B virus (HBV) and Hepatitis C virus (HCV) is recommended (level 2; Strength $C$ ).

Treatment for latent $\mathrm{TB}^{*}$ and pre-emptive therapy for chronic HBV infection are also recommended according to local 
guidelines (level 2*-5§; Strength $\mathrm{C}^{*}$ D§).

For patients with axial SpA in whom the disease cannot otherwise be controlled, TNFi may be continued throughout pregnancy (level 5; Strength D).

We strongly recommend checking the vaccination status of patients with axial SpA, following local guidelines (level 5; Strength D).

Summary of guidelines. The search identified 2 CPGs that addressed the safety of the pharmacological treatment $(15,17)$. The NICE CPG recommends monitoring of adverse events related to pharmacological treatment, according to the safety profile of each medication (15). The APLAR CPG recommends screening for TB, $\mathrm{HBV}, \mathrm{HCV}$ and HIV (in high risk populations) before starting bDMARDs and appropriate treatment, according to local guidelines (17). Treatment with TNFi is allowed during pregnancy, if clinically indicated (17). Vaccination status should be checked in each patient, according to local guidelines (17).

Recommendation/supporting evidence. NICE, APLAR $2018(15,17)$.

Evidence to recommendation. The panel agreed upon the importance of clinical and laboratory monitoring of the pharmacological treatment. Prescribing physicians should be aware of potential adverse events and contraindications related to each medication.

In patients scheduled for bDMARDs therapy, screening for TB, HBV and HCV infection must be performed. The panel did not deem necessary to suggest screening for HIV like APLAR CPG does, considering the different population to whom the Italian CPG is addressed. The panel suggests to refer to the last Italian CPG on the treatment of rheumatoid arthritis for further specifications on the screening for TB, $\mathrm{HBV}$ and $\mathrm{HCV}$ infection in patients who should start a treatment with bDMARDs (34) and underlines the importance of multidisciplinary collaboration (hepatologist, pulmonologist and infectious disease specialist) for the management of latent or active infection.
The panel agreed to suggest TNFi continuation during pregnancy, when clinically indicated. At the moment, the TNFi certolizumab pegol has received EMA approval for the treatment during pregnancy and breastfeeding, based on the results of the CRIB and CRADLE trials $(35,36)$.

Vaccination status should be checked in every patient and ideally administration of vaccines should be undertaken at least 4 weeks before starting bDMARDs. The administration of live, attenuated vaccines is absolutely contraindicated during bDMARDs treatment (34).

\section{RECOMMENDATION 12 Non-pharmacological therapy}

Patients with axial SpA should be referred to a rehabilitation specialist to start an individualized, structured exercise program. Treatment with physical therapy is recommended both in patients with active and stable axial SpA (level 1; Strength A).

In patients with spinal fusion or advanced spinal osteoporosis, treatment with spinal manipulation is not recommended (level 4; Strength C).

Summary of guidelines. The search identified 4 CPGs that addressed non-pharmacological treatment of axial $\mathrm{SpA}(12,13,15$, 17). All the CPGs recognized the need for physical therapy in patients with axial SpA. Referral to a rehabilitation specialist for patients with difficulties in everyday life activities is suggested by the NICE CPG (15). According to ACR CPG, spinal manipulation is not recommended in patients with spinal fusion or advanced spinal osteoporosis (12).

Recommendation/supporting evidence. NICE, ASAS-EULAR; ACR 2019, APLAR $2018(12,13,15,17)$.

Evidence to recommendation. The panel agreed upon the importance of specialist referral for physical therapy in patients with axial SpA. Hydrotherapy may be considered as an adjunctive therapy to manage pain and maintain or improve function for people with axial SpA. 


\section{Recommendations for monitoring of axial spondyloarthritis}

\section{RECOMMENDATION 13 Multidisciplinary collaboration}

In patients with axial $\mathrm{SpA}$, the management of musculoskeletal and extraarticular manifestations should be coordinated, as needed, between the rheumatologist and other specialists (level 5; Strength D).

Summary of guidelines. The search identified 3 CPGs that explicitly addressed the role of multidisciplinary collaboration in the management of axial $\operatorname{SpA}(13,15,16)$. Recommendation/supporting evidence. SMOLEN 2017, NICE, ASAS-EULAR $(13,15,16)$.

Evidence to recommendation. The panel agreed upon the absence of further specifications for this recommendation.

\section{RECOMMENDATION 14 Long-term complications}

Discuss risk factors for cardiovascular comorbidities with all people with axial SpA (level 5; Strength D).

Consider regular osteoporosis assessments for people with axial SpA. Advise people with axial SpA that they may be prone to fractures and should consult a healthcare professional following falls or physical trauma, particularly in the event of increased musculoskeletal pain (level 5; Strength D).

Summary of guidelines. The search identified 3 CPGs that addressed the management of long-term complications (cardiovascular events, osteoporosis) $(12,13,15)$. One CPG recommends patient education about risk factors for cardiovascular disease (15). The ACR CPG strongly recommends against instrumental screening for conduction defects and valvular heart disease (12). Screening for osteoporosis is suggested overall $(12,15)$, as well as specialist referral in case of acute spinal pain, aimed to identify any vertebral fractures $(13,15)$.

Recommendation/supporting evidence. NICE, ASAS-EULAR, ACR $2019(12,13,15)$.
Evidence to recommendation. The panel agreed upon the importance of patient education about cardiovascular risk factors, but does not recommend screening for cardiac conduction defects with electrocardiograms and screening for valvular heart disease with echocardiograms in all patients. In the context of non-pharmacological treatment and education of patients with axial SpA, smoking cessation is strongly encouraged $(13,17)$.

The panel also agreed upon the need for periodic screening for osteoporosis. Although being aware that bone mineral density measures may be elevated in spinal dual-energy X-ray absorptiometry (DEXA) due to the presence of syndesmophytes and ligamentous calcification and, therefore, that hip measurements may be more reliable, the panel suggests screening for osteoporosis with DEXA scanning of the spine as well as the hips, according to the ACR CPG (12). In evaluating results from spinal DEXA, the physician should be aware that patients with axial SpA may have an increased risk of fracture in spite of normal DEXA findings, therefore attention should be paid to acute spinal pain as a possible manifestation of a vertebral fracture.

\section{DISCUSSION}

These recommendations represent the first Italian CPG endorsed by the SIR on the management of axial SpA. Previously, in 2003, a group of Italian experts in $\mathrm{SpA}$ management suggested to refer to ASAS and SpondyloArthritis Research Consortium of Canada (SPARCC) recommendations for commencement of TNFi in patients with AS (37). In 2008, the Italian evidence-based recommendations on axial $\mathrm{SpA}$ were drafted in the context of the ' $3 \mathrm{E}$ (Evidence, Expertise, Exchange) Initiative', which however did not address the treatment with bDMARDs and referred to ASAS/EULAR CPG for this topic (38). The current recommendations were produced with the ADAPTE methodology, a systematic process of adaptation of existing CPGs which allows to collect evidence from previous research works and to adapt 
recommendations developed in another context to the local one (7). As sources for the process of adaptation on which the Italian CPG was based, only CPGs published in the previous 5 years (2014-2019) were included, because the recent introduction of new treatments for axial SpA could invalidate previous CPGs. When different CPGs from the same society were available, the most recent update was considered, as in the case of the ACR CPGs $(12,39)$. The inclusion of latest published CPGs - as the one produced by the ACR in 2019 - provided an updated extensive literature review on the most relevant clinical questions concerning treatment, especially with bDMARDs. The Italian experts broadly endorsed new ACR recommendations on this topic, with the exception of the choice of the mechanism of action in first-line bDMARD treatment: the panel decided not to recommend one mechanism of action over another due to the lack of head-to-head comparisons between TNFi and IL-17i in bio-naïve patients with axial $\mathrm{SpA}$ (12).

The ADAPTE methodology enables to gather evidence from different CPGs. In the development of Italian recommendations, data were derived not only from international CPGs regarding the pharmacological and non-pharmacological treatment of axial SpA $(12,13,15,17)$, but also from CPGs focused on specific management aspects, such as the use of imaging in the assessment of patients with axial SpA (14) or the role of a treat-to-target strategy (16). The inclusion of these different sources allowed us to cover a wide range of topics concerning the management of axial SpA. Treatment safety was extensively assessed only by the APLAR CPG, which however was developed in a quite different context from the Italian one (17). Therefore, the panel suggested to refer to recent Italian recommendations on the management of rheumatoid arthritis to address safety concerns of treatment with bDMARDs (34).

The process of external review by a broad panel of experts (rheumatologists and other specialists involved in the management of patients with axial $\mathrm{SpA}$ ) as well as by representatives of patients and health profes- sionals provided reliable feedback on the acceptability of these recommendations in the real-life Italian context. The level of agreement of external reviewers on proposed sentences was high, reinforcing the applicability of these recommendations, especially when based only on expert opinions. The low level of evidence in support of many recommendations may represent the main limitation of this CPG, thus highlighting the need for further studies on this topic.

\section{CONCLUSIONS}

A final set of 14 recommendations was developed, which provide a broad coverage of areas involved in the management of axial SpA. The dissemination and implementation of these recommendations may improve the management of patients with axial SpA in the Italian context.

\section{Plans of update}

These recommendations were endorsed by SIR as guides only and are not intended to replace the judgement of individual clinicians, since they may not apply to all patients and all clinical situations. The SIR plans to review and update these recommendations in the future to verify whether they remain valid and capture future treatments or advances in the management of axial SpA.

\section{Disclosure statements}

M. Manara has received consultant and/or speaker fees from Novartis, Celgene, Eli Lilly; I. Prevete has no conflict of interest to declare; A. Marchesoni has received honoraria and speaker fees from Abbvie, Pfizer, MSD, UCB, Novartis, Eli Lilly; S. D'Angelo has received consultant and/or speaker fees from AbbVie, Biogen, BMS, Celgene, Lilly, MSD, Novartis, Sanofi, and UCB; A. Cauli has received honoraria for consultations and/or presentations from AbbVie, Alfasigma, BMS, Celgene, Galapagos, Sanofi Genzyme, Janssen, MSD, Novartis, Pfizer, and UCB; A. Zanetti has no conflict of interest to declare; A. Ariani has received honoraria from 
Novartis, Amgen, BMS and Sanofi; A. Bortoluzzi has no conflict of interest to declare; S. Parisi has received fees from Novartis, Lilly, UCB, MSD, Pfizer, Abbvie, Biogen, Amgen, Jansenn CILAG; C. Scirè and N. Ughi have no conflict of interest to declare.

\section{Acknowledgements}

The Authors acknowledge all the external reviewers (in alphabetical order those who gave consent for publication): F. Caso, M. Castagnetti, A. Celano, MS. Chimenti, A. Delle Sedie, L. Costa, E. Lubrano, L. Meani, E. Miserocchi, A. Padula, C. Palazzi, FM. Perrotta, L. Punzi, R. Ramonda, S. Salvioli, R. Scarpa, R. Scrivo, F. Sensi, M. Vecchi. We are grateful to T. Solazzo as part of SIR secretariat for administrative support.

\section{Authors' Contributions}

All authors were involved in drafting the article or revising it critically for important intellectual content, and all authors approved the final version to be published. Conception and design: M.M., I.P., N.U., A.B., S.P., A.A., C.S.. Systematic review search and CPGs rating: M.M., I.P.. Analysis and discussion of the recommendations: M.M., I.P., A.C., S.D., A.M..

\section{REFERENCES}

1. Dougados M, Baeten D. Spondyloarthritis. Lancet. 2011; 377: 2127-2137.

2. Rudwaleit M, van der Heijde D, Landewe R, et al. The development of Assessment of SpondyloArthritis international Society classification criteria for axial spondyloarthritis (part II): validation and final selection. Ann Rheum Dis. 2009; 68: 777-783.

3. Rudwaleit M, van der Heijde D, Landewe R, et al. The Assessment of SpondyloArthritis International Society classification criteria for peripheral spondyloarthritis and for spondyloarthritis in general. Ann Rheum Dis. 2011; 70: 25-31.

4. van der Linden S, Valkenburg HA, Cats A. Evaluation of diagnostic criteria for ankylosing spondylitis. A proposal for modification of the New York criteria. Arthritis Rheum. 1984; 27: 361-368.

5. Rudwaleit M, Jurik AG, Hermann KG, et al. Defining active sacroiliitis on magnetic resonance imaging (MRI) for classification of axial spondyloarthritis: a consensual approach by the ASAS/OMERACT MRI group. Ann Rheum Dis. 2009; 68: 1520-1527.
6. Neerinckx B, Lories RJ. Structural disease progression in axial spondyloarthritis: still a cause for concern? Curr Rheumatol Rep. 2017; 19: 14 .

7. Fervers B, Burgers JS, Voellinger R, Brouwers M, Browman GP, Graham ID, et al. The ADAPTE collaboration. Guideline adaptation: an approach to enhance efficiency in guideline development and improve utilization. BMJ Qual Saf. 2011; 20: 228-236.

8. Manara M, Ughi N, Ariani A, et al. Providing updated guidelines for the management of rheumatic diseases in Italy with the ADAPTE methodology: a project by the Italian Society for Rheumatology. Reumatismo. 2019; 71(S1): 1-4.

9. Brouwers MC, Kho ME, Browman GP, et al. AGREE II: advancing guideline development, reporting and evaluation in health care. CMAJ. 2010; 182: E839-842.

10. Oxford Centre for Evidence-based Medicine. Levels of Evidence; March 2009. Available from: http://www.cebm.net/oxford-centre-evidencebased-medicine-levels-evidence-march-2009/

11. Brouwers MC, Kerkvliet K, Spithoff K. The AGREE Reporting Checklist: a tool to improve reporting of clinical practice guidelines. BMJ. 2016; 352: i1152.

12. Ward MM, Deodhar A, Gensler LS, et al. 2019 Update of the American College of Rheumatology/Spondylitis Association of America/ Spondyloarthritis Research and Treatment Network Recommendations for the Treatment of Ankylosing Spondylitis and Nonradiographic Axial Spondyloarthritis. Arthritis Rheumatol 2019; 71: 1599-1613.

13. van der Heijde D, Ramiro S, Landewé R, et al. 2016 Update of the ASAS-EULAR management recommendations for axial spondyloarthritis. Ann Rheum Dis. 2017; 76: 978-991.

14. Mandl P, Navarro-Compán V, Terslev L, et al. EULAR recommendations for the use of imaging in the diagnosis and management of spondyloarthritis in clinical practice. Ann Rheum Dis. 2015; 74: 1327-1339.

15. National Institute for Health and Care Excellence (UK). Spondyloarthritis in over 16s: diagnosis and management. London: National Institute for Health and Care Excellence (UK); 2017 Feb.

16. Smolen JS, Schöls M, Braun J, et al. Treating axial spondyloarthritis and peripheral spondyloarthritis, especially psoriatic arthritis, to target: 2017 Update of Recommendations by an International Task Force. Ann Rheum Dis. 2018; 77: 3-17.

17. Tam LS, Wei JC, Aggarwal A, et al. 2018 APLAR axial spondyloarthritis treatment recommendations. Int J Rheum Dis. 2019; 22: 340-356.

18. Braun J, Baraliakos X, Kiltz U, et al. Classification and diagnosis of axial spondyloarthri- 
tis--what is the clinically relevant difference? J Rheumatol. 2015; 42: 31-38.

19. Dougados M, Linden S, Juhlin R, et al. The European Spondylarthropathy Study Group preliminary criteria for the classification of spondylarthropathy. Arthritis Rheum. 1991; 34: 1218-1227.

20. Kellgren JH. Diagnostic criteria for population studies. Bull Rheum Dis. 1962; 13: 291-292.

21. Maksymowych WP, Lambert RG, Østergaard M, et al. MRI lesions in the sacroiliac joints of patients with spondyloarthritis: an update of definitions and validation by the ASAS MRI Working Group. Ann Rheum Dis. 2019; 78: 1550-1558.

22. Bray TJP, Jones A, Bennett AN, et al. Recommendations for acquisition and interpretation of MRI of the spine and sacroiliac joints in the diagnosis of axial spondyloarthritis in the UK. Rheumatology (Oxford). 2019; 58: 1831-1838.

23. Marchesoni A, D’Angelo S, Anzidei M, et al. Radiologist-rheumatologist multidisciplinary approach in the management of axial spondyloarthritis: a Delphi consensus statement. Clin Exp Rheumatol. 2019; 37: 575-584.

24. Wanders A, Heijde DV, Landewé R, et al. Nonsteroidal antiinflammatory drugs reduce radiographic progression in patients with ankylosing spondylitis: a randomized clinical trial. Arthritis Rheum. 2005; 52: 1756-1765.

25. Sieper J, Listing J, Poddubnyy D, et al. Effect of continuous versus on-demand treatment of ankylosing spondylitis with diclofenac over 2 years on radiographic progression of the spine: results from a randomised multicentre trial (ENRADAS). Ann Rheum Dis. 2016; 75: 1438-1443.

26. Khanna Sharma S, Kadiyala V, Naidu G, Dhir V. A randomized controlled trial to study the efficacy of sulfasalazine for axial disease in ankylosing spondylitis. Int J Rheum Dis. 2018; 21: 308-314.

27. van der Heijde D, Deodhar A, Wei JC, et al. Tofacitinib in patients with ankylosing spondylitis: a phase II, 16-week, randomised, placebo-controlled, dose-ranging study. Ann Rheum Dis 2017; 76: 1340-1347.

28. Nuovo Documento Congiunto su norma biologici biosimilari contenuta nell' art. 1 comma 407 della Legge n. 232/2016 alla luce del Secondo Position Paper AIFA; 4 maggio 2018. Available from: https:/www.reumatologia.it/ obj/files/Biosimiliari/DocumentoIntersocietario-BIO18.pdf

29. Lie E, Lindstrom U, Zverkova-Sandstrom T, et al. Tumour necrosis factor inhibitor treatment and occurrence of anterior uveitis in ankylosing spondylitis: results from the Swedish biologics register. Ann Rheum Dis. 2017; 76: 1515-1521.

30. Dick AD, Tugal-Tutkun I, Foster S, et al. Secukinumab in the treatment of noninfectious uveitis: results of three randomized, controlled clinical trials. Ophthalmology 2013; 120: 777-787.

31. Gao X, Wendling D, Botteman MF, et al. Clinical and economic burden of extra-articular manifestations in ankylosing spondylitis patients treated with anti-tumor necrosis factor agents. J Med Econ. 2012; 15: 1054-1063.

32. Hueber W, Sands BE, Lewitzky S, et al. Secukinumab, a human anti-IL-17A monoclonal antibody, for moderate to severe Crohn's disease: unexpected results of a randomised, double-blind placebo-controlled trial. Gut. 2012; 61: 1693-1700.

33. Targan SR, Feagan BG, Vermeire S, et al. A randomized, double-blind, placebo-controlled study to evaluate the safety, tolerability, and efficacy of AMG 827 in subjects with moderate to severe Crohn's disease. Gastroenterology. 2012; 143: e26.

34. Parisi S, Bortoluzzi A, Sebastiani GD, et al. The Italian Society for Rheumatology clinical practice guidelines for rheumatoid arthritis. Reumatismo. 2019; 71(S1): 22-49.

35. Mariette X, Forger F, Abraham B, et al. Lack of placental transfer of certolizumab pegol during pregnancy: results from CRIB, a prospective, postmarketing, pharmacokinetic study. Ann Rheum Dis. 2018; 77: 228-233.

36. Clowse ME, Forger F, Hwang C, et al. Minimal to no transfer of certolizumab pegol into breast milk: results from CRADLE, a prospective, postmarketing, multicentre, pharmacokinetic study. Ann Rheum Dis. 2017; 76: 1890-1896.

37. Olivieri I, Salvarani C, Cantini F, et al. Recommendations for starting anti TNF-alpha in patients with ankylosing spondylitis. Reumatismo. 2003; 55: 220-223.

38. D'Angelo S, Padula A, Nigro A, et al. Italian evidence-based recommendations for the management of ankylosing spondylitis: the $3 \mathrm{E}$ Initiative in Rheumatology. Clin Exp Rheumatol. 2008; 26: 1005-1011.

39. Ward MM, Deodhar A, Akl EA, et al. American College of Rheumatology/Spondylitis Association of America/Spondyloarthritis Research and Treatment Network 2015 recommendations for the treatment of ankylosing spondylitis and nonradiographic axial spondyloarthritis. Arthritis Rheumatol. 2016; 68: 282-298. 